\title{
Home- vs. Laboratory-Based Management Of OSA: An Economic Review
}

\author{
$\mathrm{Ken} \mathrm{He}^{1} \cdot$ Richard Kim ${ }^{1,2}$ • Vishesh K. Kapur ${ }^{1}$
}

Published online: 12 April 2016

(C) Springer International Publishing AG 2016

\begin{abstract}
Obstructive sleep apnea (OSA) is a common condition that impairs quality of life and health. Diagnosis and treatment of OSA is cost-effective; however, the economics of various management strategies remain to be defined. Home sleep apnea tests (HSAT) provide an alternative to laboratory based polysomnography (PSG) and are less expensive than PSG on a per test basis; however, when utilized within a framework that has been demonstrated to provide comparable clinical outcomes, home testing pathways incur additional costs to compensate for failed studies and lower diagnostic accuracy. A cost-minimization analysis from a randomized controlled trial showed that the cost advantage of a home management pathway narrowed significantly when these additional costs are considered. Further, when the actual costs of providing HSAT rather than what is reimbursed by insurance were considered, the cost advantage was further attenuated. A comprehensive cost-effectiveness analysis (CEA), favored a lab over a home approach based on modeling that projected
\end{abstract}

This article is part of the Topical Collection on Sleep Related Breathing Disorders

Vishesh K. Kapur

vkapur@uw.edu

Ken $\mathrm{He}$

kenhe@uw.edu

Richard Kim

rdkim@uw.edu

1 Division of Pulmonary and Critical Care Medicine, University of Washington, UW Medicine Sleep Center, 325 Ninth Ave, Box 359803, Seattle, WA 98104, USA

2 Hutchinson Institute for Cancer Outcomes Research, Fred Hutchinson Cancer Research Institute, 1100 Fairview Ave N., M3-B232, Seattle, WA 98109, USA that the costs of erroneous diagnosis over a long time span for the home approach outweighed lower test costs. Studies have identified the following factors that influence costeffectiveness of home-based management: cost of untreated OSA, prevalence of OSA, performance characteristics of the selected test, time horizon, and whether backup PSG is used for failed HSAT. More clinical studies are needed to provide the inputs for more robust CEA regarding this issue.

Keywords Sleep apnea $\cdot$ Economics $\cdot$ Cost-effectiveness · Polysomnography $\cdot$ Home sleep testing

$\begin{array}{ll}\text { Abbreviations } \\ \text { AASM } & \text { American Academy of Sleep Medicine } \\ \text { AHI } & \text { Apnea-hypopnea index } \\ \text { APAP } & \text { Auto-titrating positive airway pressure } \\ \text { CEA } & \text { Cost-effectiveness analysis } \\ \text { HSAT } & \text { Home sleep apnea testing } \\ \text { ICER } & \text { Incremental cost-effectiveness ratio } \\ \text { OSA } & \text { Obstructive sleep apnea } \\ \text { PAP } & \text { Positive airway pressure } \\ \text { PSG } & \text { Polysomnography } \\ \text { RCT } & \text { Randomized controlled trial } \\ \text { QALY } & \text { Quality-adjusted life year } \\ \text { QOL } & \text { Quality of life }\end{array}$

\section{Introduction}

Obstructive sleep apnea (OSA) is common, and when untreated, is associated with reduced quality of life, work productivity loss, motor vehicle accidents, and risk of cardiovascular disease [1-3]. Conventionally, diagnosis and treatment of OSA involves overnight laboratory-based diagnostic 
polysomnography (PSG) followed by a second night of positive airway pressure (PAP) titration PSG. This process, considered the gold standard method for diagnosis and initiation of PAP therapy, is time and labor intensive, and therefore costly [4-6]. Additionally, patients are inconvenienced by travel to testing sites, returning for a second night, and sleeping in an unfamiliar environment. In some settings, the availability of laboratory testing may be limited and this can lead to delays in care. For some patients, it is not technically feasible to conduct studies in the sleep center due to health conditions and limited mobility. The utilization of split-night protocols whereby diagnostic and PAP titration PSG occur on the same night alleviates some burdens associated with traditional testing with similar effectiveness [7-9].

Home sleep apnea tests (HSAT) offer the opportunity to improve access to care, simplify diagnosis of OSA, and reduce costs. The American Academy of Sleep Medicine (AASM) recommends that HSAT only be used in patients with high pretest probability of moderate to severe disease without other significant medical or sleep related comorbidities under the guidance of a sleep specialist within a management pathway that uses PSG to confirm diagnosis when HSAT findings are inconclusive or negative [10]. Use of auto-titrating positive airway pressure (APAP) devices can also streamline management of OSA by obviating the need to perform attended PSG PAP titration in some OSA patients [11]. Studies have shown equivalent outcomes using APAP compared to manual PAP titration in appropriately selected patients; medically stable patients at high risk for moderate to severe OSA who do not have significant cardiorespiratory comorbidities [12-16]. Combining HSAT and APAP, it is possible to construct a home-based OSA management strategy as an alternative to lab-based PSG and titration. Several recent randomized clinical trials (RCT) demonstrate non-inferior outcomes using home-based approaches [17-19, 20••]. The HomePAP study, a large multicenter trial with 373 participants, showed noninferior treatment adherence, PAP therapy acceptance, treatment pressures, and functional improvements at 3 months for the home arm $[20 \bullet \cdot]$.

OSA patients consume significant healthcare resources, and this results in an annual economic burden estimated to be in the billions of dollars just in the USA alone [21-23]. Costeffectiveness analysis (CEA), a technique that provides a framework for assessing value for money spent among alternative management strategies, shows that treatment of OSA with PAP is cost-effective compared to no treatment [22-24]. For example, one CEA showed societal incremental costeffectiveness ratio (ICER) of \$314 per quality-adjusted life year (QALY) gained which compares very favorably to other large, publically funded health interventions based on a willingness to pay a threshold of $\$ 50,000$ per QALY $[24,25]$. The economics of home- relative to lab-based management of OSA is of considerable interest given the significant costs associated with the disorder as well as its diagnosis and treatment. We review recent literature to elucidate what is known on this topic (refer to Table 1 for summary of studies).

\section{Cost Effectiveness Analyses}

A potential advantage of the home-based diagnosis and treatment of OSA is lower resource utilization and lower costs [35, 36]. However, the most comprehensive CEA of this question found that a full night PSG followed by titration was the most cost-effective strategy, compared with split-night PSG and HSAT/APAP [26••]. In this analysis, both the split-night PSG and HSAT/APAP strategies had full night/titration backup. The analysis was taken from a third party payer perspective using Markov modeling over a lifetime horizon. The base model was composed of 50-year-old men with a $50 \%$ pretest probability of moderate to severe OSA (AHI $\geq 15)$. Key assumptions made in this study include rates of patient attrition, PAP acceptance/ adherence, and quality of life (QOL) reduction for those on PAP due to a wrong diagnosis. Additional structural assumptions made in the model include the following: PAP discontinuation remaining in effect for the remaining time horizon, lack of attrition after diagnostic PSG, lack of PSG/titration failures, increased number of clinic follow-ups for HSAT/APAP pathway, and no change in probability of stopping PAP based on the accuracy of diagnosis (person with wrong OSA diagnosis was assumed to have the same probability of stopping PAP as correct OSA diagnosis). These assumptions favor the strategy with superior test performance (full night PSG and titration pathway) especially over the lifetime horizon, because costs related to wrong diagnoses persist over the entire time horizon. The CEA did find that all testing methods were cost-effective at a threshold of \$50,000 per QALY gained.

More recent CEAs have been more limited in scope; one modeled cost-effectiveness of three diagnostic/treatment strategies (do nothing, type 3 HSAT/PAP, or OSA screening inventory followed by split-night PSG/PAP) in a population with low prevalence and risk for OSA (active duty military personnel) [27•]. Uniquely, this study also considered costs of lost workplace productivity, whereas the prior CEA only focused on health care utilization and medical outcomes. This study found that the home testing approach was favored over the screen-then-test approach. Backup PSG was not considered for failed HSAT and all patients with AHI $>5$ proceeded to PAP therapy. Factors that favored the HSAT strategy were poor diagnostic performance of the screening inventory (leading to more PSGs) and lower costs incurred from missing OSA diagnoses due to low OSA prevalence. In contrast, screen/split-night PSG was favored at longer time horizons and with better screening inventory performance. The donothing approach was favored in low prevalence, low cost, and short time horizon settings. The base model assumed untreated OSA (5\% prevalence) incurs a $20 \%$ reduction in work 


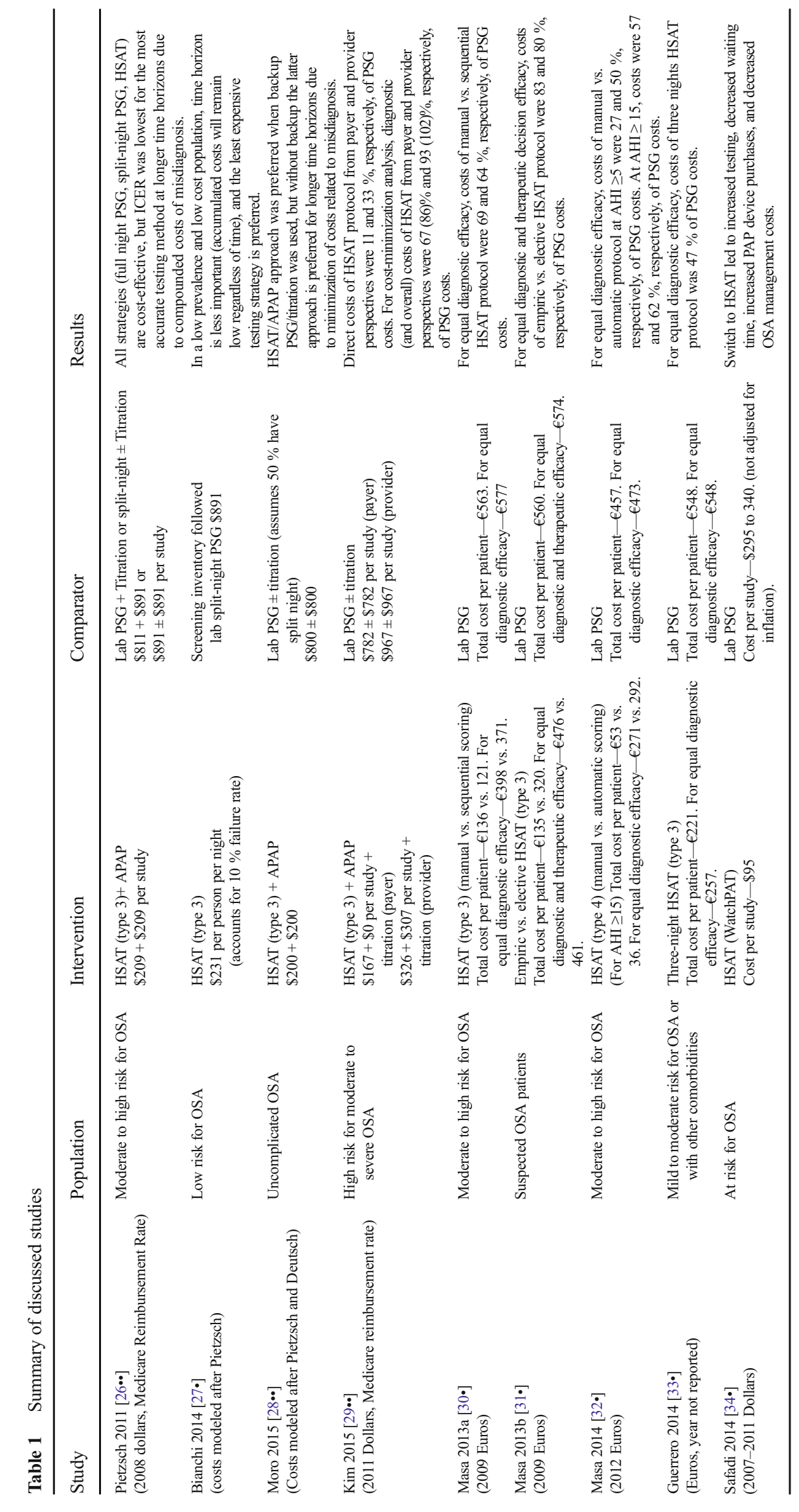


efficiency that equates to loss of $\$ 10,000$ per year based on an annual military salary of $\$ 50,000$ over 20 service years (time horizon). The balance of potential cost savings from optimal job performance due to OSA treatment relative to costs associated with OSA management was analyzed for each strategy. The HSAT approach was favored in the base model in terms of per person costs (\$4516) vs. screen/split-night PSG (\$5468). Sensitivity analyses varying cost of untreated OSA, prevalence, time horizon, and cost of HSAT in most cases also favored the HSAT approach.

In a similar vein, another recent CEA explored the effects of the following: pretest probability, cost of untreated OSA, time horizon, and using backup PSG/ titration for failed HSAT/APAP, in an uncomplicated OSA population [28••]. It evaluated direct cost and cost-effectiveness from a third party payer perspective by simulating four strategies-treat no one, treat all, PSG and titration (50\% chance of being split), or HSAT/APAP. Studies were allowed to be repeated once if initial evaluation was indeterminate. Test costs and performance (sensitivity/specificity) assumptions were extrapolated from prior studies. Higher OSA prevalence favored HSAT, but also increased chances of meeting split-night criteria, favoring cost savings with PSG. Low OSA prevalence and low cost of untreated OSA favored the treat-no-one approach, whereas high OSA prevalence and high cost of untreated OSA favored the treat-all approach. If downstream consequences of missed OSA diagnoses and its associated costs were neglected and time horizon was short, the cheapest strategy is always preferred (i.e., no testing). HSAT/APAP approach was preferred when backup PSG/titration was used, but without backup, the PSG approach was preferred for longer time horizons due to minimization of costs related to misdiagnoses.

\section{Randomized Controlled Trial-Based Cost Analysis}

In contrast to the prior CEAs, a recent study performed a cost-minimization analysis using results from the HomePAP study and thus did not need to make assumptions regarding PAP acceptance, patient attrition, failed tests, and rates of successful titrations [29••]. This study was also unique in that it considered costs from a provider perspective in addition to third party payer perspective (as was considered in the prior CEAs); in other words, the resource inputs reflected in labor and capital costs of each OSA management pathway to the provider were estimated over a 1-year time horizon. The findings mirror cost-effectiveness since the trial demonstrated equivalent outcomes in home vs. lab strategies. A limitation of the trial design was that patients with $\mathrm{AHI}$
$<15$ after diagnostic testing were excluded. To mimic a more realistic scenario, a model in which patients with AHI $\geq 5$ were treated was also developed. Home program was always cheaper in all scenarios for the payer (\$1575 vs. $\$ 1840$ for the base case) in part due to higher cost of PSG and no reimbursement for APAP, but the gap shrank due to split-night PSG, backup PSG/titration for failed HSAT/APAP, higher number of failed HSAT/APAP, and larger number of patients treated with PAP. While for the provider, costs (capital, labor, overhead) were generally less for the home program, this was not true for all scenarios. Another important finding was that provider operating margin (payer cost or "reimbursement" minus provider cost) was negative for home program in all scenarios as Medicare reimbursement levels for HSAT are lower than provider costs. This disparity between home-based cost perspectives suggests that high quality HSAT programs may be unsustainable and potential lower quality (and possibly worse outcomes) programs may result as providers are forced to cut costs. The provider perspective highlighted the large number of cost components necessary to ensure high quality home-based OSA management, which narrowed the cost difference relative to lab management.

\section{Equivalency Trials}

A series of studies from Spain looked at cost implications under various scenarios from equivalency trials comparing outcomes in patients who underwent both HSAT and PSG [30•, 31•, 32•, 33•]. Cost data in this situation is not directly comparable because distinct testing strategies were not utilized, unlike in the previously discussed studies. Despite these limitations, these studies illustrate several key points about cost and outcomes between HSAT and PSG.

The first study looked at costs associated with achieving equivalent diagnostic efficiency comparing different HSAT scoring methodologies (manual vs. sequential scoring) vs. PSG and found that an individual HSAT is substantially less costly to perform (with some minor additional saving if not all HSAT studies are manually scored), but cost to achieve diagnostic equivalence with PSG results in less dramatic savings [30•]. There was better AHI agreement between PSG and manually scored HSAT compared to automated scoring. Total cost to reach diagnosis of OSA per patient in sequential and manual HSAT groups were 22 and $24 \%$ of PSG, respectively. However, to achieve equal diagnostic efficacy, HSAT costs were 64 and $69 \%$ of PSG for sequential and manual HSAT scoring, respectively. A second study, based on data from the same clinical trial as the first, indicated that empiric (test all patients) vs. elective (test patients at high clinical 
probability of OSA) HSAT with backup PSG resulted in similar cost savings relative to $\mathrm{PSG}$ to achieve equivalent diagnostic and therapeutic efficacy [31•]. Elective HSAT dramatically reduced the number of HSATs while only slightly increasing the number of backup PSGs required compared to empiric HSAT. Empiric vs. elective HSAT resulted in 24 and $57 \%$ of PSG costs, respectively. For equal diagnostic and treatment efficacy, empiric and elective HSAT resulted in 83 and $80 \%$ of PSG approach costs, respectively. Since elective HSAT had more appropriately selected high clinical probability patients that go on to diagnosis and treatment, there were less indeterminate results necessitating backup PSGs. Similarly, more backup PSGs were needed to achieve diagnostic and therapeutic efficacy in the empiric HSAT arm due to indeterminate and false positive/negative studies leading to higher costs.

Analysis of data from a Spanish multicenter, crossover study of adults at moderate to high risk for OSA concluded that manual scoring is less costly than automated scoring due to relatively better diagnostic efficacy than automated scoring at lower AHI levels [32•]. Though the total cost per test relative to PSG for automated (8\%) scoring was less than for manual scoring (12\%), to achieve equal diagnostic efficacy, HSAT costs for OSA diagnosis (AHI $\geq 15$ ) was higher for automated scoring $(62 \%)$ than for manual scoring $(57 \%)$. At $\mathrm{AHI} \geq 5$, manual scoring resulted in even more cost savings relative to automatic scoring.

Finally, a Spanish trial that included patients without high risk of OSA, found that nothing was gained from extra nights of consecutive testing besides providing a fail-safe from invalid recordings on first night recording and that best treatment decision concordance was among sleep specialists who also tended to recommend more conservative treatment measures (cost savings) compared to other providers (respiratory specialists and residents with some training in sleep) [33•]. Total test cost of three nights of HSAT was $40 \%$ of PSG cost and increased to $47 \%$ for equivalent diagnostic efficacy based on AHI $\geq 5$. Due to better HSAT performance characteristics (sensitivity/specificity), few backup PSGs were needed, resulting in greater savings in this study compared to the other Spanish studies reviewed.

\section{Impact on Healthcare System}

A retrospective analysis from a large Israeli health system that recently transitioned from lab- to home-based management of OSA, reported decreased diagnostic costs despite more patients being evaluated for OSA over a 4-year period [34•]. In this study, the homebased management used HSAT in uncomplicated patients with suspected OSA, but allowed PSG for those with serious comorbidities unsuited for HSAT. When data from 2007-2008 (100\% PSG) was compared to 2010-2011 (24\% PSG), there was a $90 \%$ increase in the number of sleep studies performed (1471 vs. 2794), an increase in CPAP purchases (597 vs. 831), and a decrease in waiting times for any sleep study (9.9 vs. 1.1 weeks). Patients surveyed prospectively in a random sample reported equivalent satisfaction, less discomfort with HSAT, and a preference for HSAT. Despite more testing during 2010-2011, overall total direct costs of testing from third party payer perspective were $20 \%$ less due to increased utilization of HSAT (\$95) vs. PSG (\$295-340). This study does not address the effectiveness of the new strategy from a societal perspective and the relative cost-effectiveness of the new strategy vs the prior one. Factors such as the impact of misdiagnoses and their consequences could impact relative costeffectiveness of the two strategies.

\section{Conclusion}

The availability of a home-based strategy for management of OSA is transforming the practice of sleep medicine. Evidence from RCT supporting that a home-based pathway can achieve comparable outcomes to a labbased pathway in select clinical populations along with the potential for reduced costs have influenced payers to allow and, in some cases mandate, use of a home-based strategy. However, the economic consequences of home testing strategies remain to be adequately evaluated.

The cost of a single HSAT is unequivocally lower than a PSG, though this cost advantage is reduced by additional costs incurred to compensate for the reduced accuracy of HSAT. Further clouding a comprehensive economic evaluation is the potential for inferior clinical outcomes with HSAT. Important factors in determining the cost-effectiveness of home management of OSA include prevalence of OSA, clinical characteristics of the patient population, performance characteristics of HSAT, details of the management pathway such as whether backup PSG is used for failed HSAT, the time horizon over which costs are considered, and the cost of untreated OSA.

Moving forward, additional data from traditional randomized controlled trials as well as data from real-world studies that span longer time horizons and capture clinical effectiveness and cost data are needed to clarify the economic impact of HSAT. Data from these types of studies will allow more robust CEA by minimizing modeling assumptions, and help identify factors that will optimize cost-effectiveness of a home-based management of OSA. 


\section{Compliance with Ethical Standards}

Conflict of Interest Ken He, Richard Kim, and Vishesh K. Kapur declare that they have no conflict of interest.

Human and Animal Rights and Informed Consent This article does not contain any studies with human or animal subjects performed by any of the authors.

\section{References}

Papers of particular interest, published recently, have been highlighted as:

- Of importance

- Of major importance

1. Young T, Peppard PE, Gottlieb DJ. Epidemiology of obstructive sleep apnea: a population health perspective. Am J Respir Crit Care Med. 2002;165(9):1217-39.

2. Peppard PE, Young T, Barnet JH, et al. Increased prevalence of sleep-disordered breathing in adults. Am J Epidemiol. 2013;177(9):1006-14.

3. Punjabi NM. The epidemiology of adult obstructive sleep apnea. Proc Am Thorac Soc. 2008;5(2):136-43.

4. Epstein LJ, Kristo D, Strollo Jr PJ, et al. Clinical guideline for evaluation, management, and long-term care of obstructive sleep apnea in adults. J Clin Sleep Med. 2009;5(3):263-76.

5. Hossain JL, Shapiro CM. The prevalence, cost implications, and management of sleep disorders: an overview. Sleep Breath. 2002;6(2):85-102.

6. Flemons WW, Douglas NJ, Kuna ST, et al. Access to diagnosis and treatment of patients with suspected sleep apnea. Am J Respir Crit Care Med. 2004;169(6):668-72.

7. BaHammam AS, ALAnbay E, Alrajhi N, Olaish AH. The success rate of split-night polysomnography and its impact on continuous positive airway pressure compliance. Ann Thorac Med. 2015;10(4):274-8.

8. Khawaja IS, Olson EJ, van der Walt C, et al. Diagnostic accuracy of split-night polysomnograms. J Clin Sleep Med. 2010;6(4):357-62.

9. Deutsch PA, Simmons MS, Wallace JM. Cost-effectiveness of splitnight polysomnography and home studies in the evaluation of obstructive sleep apnea syndrome. J Clin Sleep Med. 2006;2(2):145-53.

10. Collop N, Anderson WM, Boehlecke B, et al. Clinical guidelines for the use of unattended portable monitors in the diagnosis of obstructive sleep apnea in adult patients. J Clin Sleep Med. 2007;3(7):737-47.

11. Bierer GB, Ryden A, Aysola RS. Advanced positive airway pressure modalities. Curr Sleep Med Rep. 2015;1:257-64.

12. Kushida CA, Berry RB, Blau A, et al. Positive airway pressure initiation: a randomized controlled trial to assess the impact of therapy mode and titration process on efficacy, adherence, and outcomes. Sleep. 2011;34(8):1083-92.

13. Nolan GM, Doherty LS, Mc Nicholas WT. Auto-adjusting versus fixed positive pressure therapy in mild to moderate obstructive sleep apnea. Sleep. 2007;30(2):189-94.

14. Mulgrew AT, Cheema R, Fleetham J, Ryan CF, Ayas NT. Efficacy and patient satisfaction with autoadjusting CPAP with variable expiratory pressure vs standard CPAP: a two-night randomized crossover trial. Sleep Breath. 2007;11(1):31-7.
15. Fietze I, Glos M, Moebus I, Witt C, Penzel T, Baumann G. Automatic pressure titration with APAP is as effective as manual titration with CPAP in patients with obstructive sleep apnea. Respiration. 2007;74(3):279-86.

16. Morgenthaler TI, Aurora RN, Brown T, Standards of Practice Committee of the AASM, et al. American Academy of Sleep Medicine. Practice parameters for the use of autotitrating continuous positive airway pressure devices for titrating pressures and treating adult patients with obstructive sleep apnea syndrome: an update for 2007. An American Academy of Sleep Medicine report. Sleep. 2008;31(1):141-7.

17. Skomro RP, Gjevre J, Reid J, et al. Outcomes of home-based diagnosis and treatment of obstructive sleep apnea. Chest. 2010;138(2): 257-63.

18. Kuna ST, Gurubhagavatula I, Maislin G, et al. Noninferiority of functional outcome in ambulatory management of obstructive sleep apnea. Am J Respir Crit Care Med. 2011;183(9): 1238-44.

19. Berry RB, Hill G, Thompson L, et al. Portable monitoring and autotitration versus polysomnography for the diagnosis and treatment of sleep apnea. Sleep. 2008;31(10):1423-31.

20.• Rosen CL, Auckley DA, Benca R, et al. A multisite randomized trial of portable sleep studies and positive airway pressure autotitration versus laboratory-based polysomnography for the diagnosis and treatment of obstructive sleep apnea: the HomePAP study. Sleep. 2012;35(6):757-67. This randomized trial showed that home-based diagnosis and treatment of OSA was non-inferior to lab-based PSG and PAP titration when carried out in the context of an appropriate clinical population and management pathway.

21. Kapur V, Blough DK, Sandblom RE, Hert R, de Maine JB, Sullivan $\mathrm{SD}$, et al. The medical cost of undiagnosed sleep apnea. Sleep. 1999;22(6):749-55.

22. Tarasiuk A, Reuveni H. The economic impact of obstructive sleep apnea. Curr Opin Pulm Med. 2013;19(6):639-44.

23. Kapur VK. Obstructive sleep apnea: diagnosis, epidemiology, and economics. Respir Care. 2010;55(9):1155-67.

24. Ayas NT, FitzGerald JM, Fleetham JA, et al. Cost-effectiveness of continuous positive airway pressure therapy for moderate to severe obstructive sleep apnea/hypopnea. Arch Intern Med. 2006;166(9): 977-84.

25. Neumann PJ, Cohen JT, Weinstein MC. Updating cost-effectiveness - the curious resilience of the $\$ 50,000$-per-QALY threshold. N Engl J Med. 2014;371(9):796-7.

26.• Pietzsch JB, Garner A, Cipriano LE, et al. An integrated health-economic analysis of diagnostic and therapeutic strategies in the treatment of moderate-to-severe obstructive sleep apnea. Sleep. 2011;34(6):695-709. This cost-effectiveness analysis modeled different OSA diagnostic and treatment strategies, and showed full-night PSG with CPAP therapy was most cost-effective.

27. Bianchi MT, Hershman S, Bahadoran M, et al. The challenge of undiagnosed sleep apnea in low-risk populations: a decision analysis. Mil Med. 2014;179(8 Suppl):47-54. This decision analysis modeled different OSA diagnostic strategies in a low-risk military population and showed HSAT in all comers as the preferred strategy over screening inventory followed by PSG

28.• Moro M, Westover MB, Kelly J, et al. Decision modeling in sleep apnea: the critical roles of pre-test probability, cost of untreated OSA, and time horizon. J Clin Sleep Med. 2015;12:409-18. This decision analysis modeled different OSA diagnostic and treatment strategies, and showed how OSA related factors influenced the best strategy. The optimal diagnostic approach to 
uncomplicated OSA depends on pre-test probability, cost of untreated OSA, and time horizon.

29.• Kim RD, Kapur VK, Redline-Bruch J, et al. An economic evaluation of home versus laboratory-based diagnosis of obstructive sleep apnea. Sleep. 2015;38(7):1027-37. This cost-minimization analysis based on HomePAP study data showed that for payers, a home-based diagnostic pathway for obstructive sleep apnea with robust patient support incurs fewer costs than a laboratory-based pathway. For providers, costs are comparable if not higher, resulting in a negative operating margin.

30. Masa JF, Garcia-Ledesma E, Pereira R, et al. Effectiveness of sequential automatic-manual home respiratory polygraphy scoring. Eur Respir J. 2013;41(4):879-87. Sequential (automatic followed by manual) scoring of home sleep apnea tests is a good alternative with lower direct costs for equivalent diagnostic efficacy compared to manually scored home sleep apnea tests or lab PSG.

31. Masa JF, Corral J, Pereira R, et al. Effectiveness of three sleep apnea management alternatives. Sleep. 2013;36(12):1799-807. HSAT is a less costly than PSG for equivalent diagnosis and therapeutic decision making capability in patients with suspected obstructive sleep apnea. There is no cost advantage in using HSAT for all patients compared to HSAT for the most symptomatic patients and PSG for the rest.

32. Masa JF, Duran-Cantolla J, Capote F, et al. Effectiveness of home single-channel nasal pressure for sleep apnea diagnosis. Sleep.
2014;37(12):1953-61. HSAT with single-channel nasal pressure sensor is less costly than PSG to achieve same level of diagnostic efficacy. Manual scoring is less costly than automatic scoring to achieve equivalent diagnostic efficacy.

33. Guerrero A, Embid C, Isetta V, et al. Management of sleep apnea without high pretest probability or with comorbidities by three nights of portable sleep monitoring. Sleep. 2014;37(8):1363-73. Three consecutive nights of HSAT in patients at lower risk of OSA or with comorbid diseases had lower direct costs compared to lab PSG to achieve equivalent diagnostic efficacy. The best agreement of therapeutic decisions was achieved by the sleep medicine specialists (versus non-sleep medicine providers).

34. Safadi A, Etzioni T, Fliss D, et al. The effect of the transition to home monitoring for the diagnosis of OSAS on test availability, waiting time, patients' satisfaction, and outcome in a large health provider system. Sleep Disord. 2014;2014:418246. There was an increased number of sleep tests, decreased waiting time, similar patient satisfaction, and decreased direct costs for a third party payer after transition from lab-based to home-based testing.

35. Andreu AL, Chiner E, Sancho-Chust JN, et al. Effect of an ambulatory diagnostic and treatment programme in patients with sleep apnoea. Eur Respir J. 2012;39(2):305-12.

36. Dingli K, Coleman EL, Vennelle M, et al. Evaluation of a portable device for diagnosing the sleep apnoea/hypopnoea syndrome. Eur Respir J. 2003;21(2):253-9. 